\title{
Gas/oil/water flow measurement by electrical capacitance tomography
}

\author{
Yi Li ${ }^{1}$, Wuqiang Yang ${ }^{1,3}$, Cheng-gang $\mathrm{Xie}^{2}$, Songming Huang ${ }^{2}$, \\ Zhipeng $\mathrm{Wu}^{1}$, Dimitrios Tsamakis ${ }^{1}$ and Chris Lenn ${ }^{2}$ \\ ${ }^{1}$ School of Electrical and Electronic Engineering, The University of Manchester, Sackville Street, \\ Manchester M13 9PL, UK \\ ${ }^{2}$ Schlumberger Gould Research, High Cross, Madingley Road, Cambridge CB3 0EL, UK \\ E-mail: w.yang@manchester.ac.uk and cxie@slb.com
}

Received 22 August 2012, in final form 31 October 2012

Published DD MM 2012

Online at stacks.iop.org/MST/23/000000

\begin{abstract}
In the oil industry, it is important to measure gas/oil/water flows produced from oil wells. To determine oil production, it is necessary to measure the water-in-liquid ratio (WLR), liquid fraction and some other parameters, which are related to multiphase flow rates. A research team from the University of Manchester and Schlumberger Gould Research have developed an experimental apparatus for gas/oil/water flow measurement based on a flow-conditioning device and electrical capacitance tomography (ECT) and microwave sensors. This paper presents the ECT part of the developed apparatus, including the re-engineering of an ECT sensor and a model-based image reconstruction algorithm, which is used to derive the WLR and the thickness of the liquid layer in oil-continuous annular flows formed by the flow-conditioning device. The ECT sensor was tested both at Schlumberger and on TUV-NEL's Multiphase Flow Facility. The experimental results are promising.
\end{abstract}

Keywords: multiphase flow measurement, gas/oil/water flows, water-in-liquid ratio, liquid fraction, electrical capacitance tomography

Q1 (Some figures may appear in colour only in the online journal)

\section{Introduction}

In the oil industry, it is important to make an online measurement of gas/oil/water flows, using multiphase flow meters (MPFMs) (Xie et al 2007). To obtain the production flow rates of oil and produced water, it is necessary to measure, directly and/or indirectly, the water-in-liquid ratio (WLR), gas or liquid fraction and phase velocities. A number of MPFMs have been developed by research organizations and companies, with different measurement technologies. While those MPFMs are commercially available, there are still some challenging problems, such as the sensitivity of phase-fraction measurements to variations in multiphase flow regimes and in the fluid properties. Therefore, multiphase flow measurement is still an ongoing research topic, aiming to improve the robustness of the online measurement of unprocessed oil well

\footnotetext{
3 Author to whom any correspondence should be addressed.
}

streams, to monitor continuous production of each oil well and to provide a low-cost MPFM solution, which is especially needed for marginal-field production monitoring. Some other applications of MPFMs include production optimization, flow assurance, well testing, production allocation metering, fiscal or custody transfer measurements and wet gas (Norwegian Society for Oil and Gas Measurement 2005).

Multiphase flow is a complex phenomenon, which is still difficult to fully understand, predict, model and measure accurately. The multiphase flow regime varies, depending on operating conditions, fluid properties, flow rates and the orientation and geometry of the pipe through which the fluids flow. The spatial and/or temporal distribution of the fluid phases differs for various flow regimes, and is usually beyond human control, although the transition between different flow regimes may be a gradual process. In terms of gas and liquid superficial velocities, naturally occurring (fully developed) flow regimes can be grouped into dispersed flow, separated flow, intermittent flow or a combination of these as the physical 
parameters, e.g. density, viscosity and surface tension of the liquid phases, affect the flow regimes (Norwegian Society for Oil and Gas Measurement 2005).

Another way to broadly classify the multiphase flow regime is by the gas volume fraction $(\mathrm{GVF})$ at line pressure and temperature conditions, which is the gas volumetric flow rate divided by the gas-liquid total volumetric flow rate. This classification method is relevant to multiphase metering because a meter designed to measure gassy liquid (with a few percentage of gas) would be significantly different from one designed to measure wet gas (Rajan et al 1993).

In this research, a flow-conditioning device is used to form predominately a gas-liquid annular flow, aiming to minimize variation in flow regime over a wide range of GVFs. A flowmetering Venturi is used to increase centrifugal acceleration at its throat relative to the induced swirl at the Venturi inlet. It has been envisaged that this could enhance the displacement of the liquid to the inner pipe wall within the Venturi throat section and reduce gas entrained in the liquid, enabling a more accurate liquid flow rate measurement by measuring its physical properties, such as the WLR and the thickness (fraction/holdup) of the liquid layer.

It is well known that an oil/water flow can be oilcontinuous or water-continuous, depending on the WLR, the density and/or viscosity of oil and water, and the presence of other chemicals such as surfactant, and to some extent on the total flow rate. Although it is difficult to predict the exact phase-inversion point, it has been known that an oil-continuous flow may correspond to a WLR range as narrow as $0-30 \%$, or as broad as $0-70 \%$. In an oil-continuous case, it is possible to measure the oil/water flow by measuring capacitance. However, the problem with conventional capacitance sensors is that the capacitance measurements by such conventional sensors are flow-regime-dependent (Falcone et al 2010).

Electrical capacitance tomography (ECT) can provide the permittivity distribution of an oil-continuous flow, which is electrically non-conductive (Xie et al 1992, Yang et al 1995, Ismail et al 2005). In the case of a water-continuous flow, microwave sensors may be used to measure the dielectric properties based on transmission (Xie 2006). Therefore, it is possible to combine ECT with microwave sensors to deal with a large range of WLR. This paper will describe a method of using ECT to measure oil-continuous flows.

\section{Method}

\subsection{Principle of ECT}

ECT has been developed for imaging industrial processes containing dielectric materials (Huang et al 1989), by obtaining a permittivity distribution derived from the measured capacitance of multiple electrode pairs, where the changes in capacitance $C$ (electric charge $Q$ ) are caused by the change in permittivity distribution. The relationship between the capacitance and the permittivity distribution can be expressed by the following equation (Xie et al 1992):

$$
C=\frac{Q}{V}=-\frac{1}{V} \iint_{\Gamma} \varepsilon(x, y) \nabla \phi(x, y) \mathrm{d} \Gamma
$$

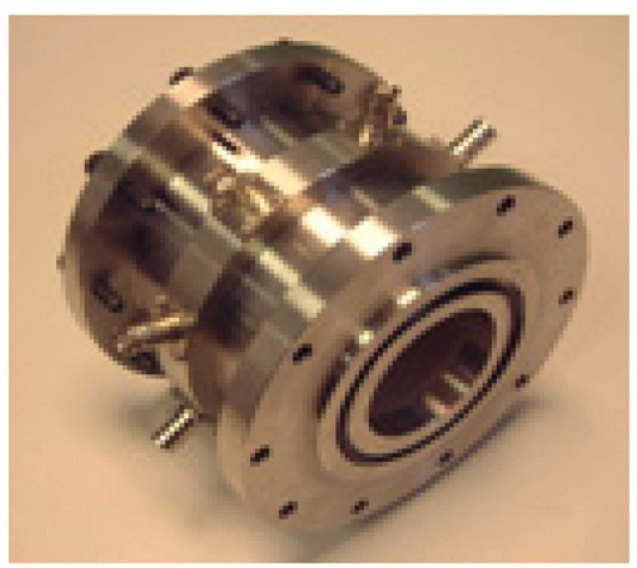

Figure 1. Photo of the eight-electrode ECT sensor.

where $\varepsilon(x, y)$ is the permittivity distribution in the sensing field, $V$ is the potential difference between two chosen sensing electrodes and $\phi(x, y)$ is the potential distribution.

A typical ECT sensor consists of 8 or 12 electrodes (Xie et al 1992) evenly mounted on an insulating liner section of a pipe. When one of the electrodes is excited in turn and other electrodes are kept at zero potential, a total of $N /(N-1) / 2$ independent capacitance measurements can be made where $N$ is the number of electrodes. For example, with an eightelectrode ECT sensor there are 28 independent capacitance measurements in total. The cross-sectional distribution of permittivity is obtained from these capacitance measurements using image reconstruction algorithms, e.g. linear back projection (LBP) (Xie et al 1992), Landweber iteration (Yang and Peng 2003) or model-based algorithm (Isaksen and Nordtved 1993).

In this work, an AC-based ECT system (Yang and York 1999) is used to take measurements from an eight-electrode ECT sensor. The data sampling rate is $\sim 100$ frames $\mathrm{s}^{-1}$. The excitation frequency is $140 \mathrm{kHz}$ and the excitation amplitude is $14 \mathrm{~V}_{\mathrm{p}-\mathrm{p}}$. The eight-electrode ECT sensor is shown in figure 1. It is made by Schlumberger Gould Research (formerly Schlumberger Cambridge Research-SCR). The measurement section is $52 \mathrm{~mm}$ in inner diameter and $100 \mathrm{~mm}$ in axial length (mainly made of 316 stainless steel). The eight electrodes are $50 \mathrm{~mm}$ in axial length and $25 \mathrm{~mm}$ in width with the electrode angular size of $\sim 42^{\circ}$. They are mounted evenly around the outside of a PEEK tube liner of $8 \mathrm{~mm}$ wall thickness (dielectric constant $\sim 3.2$ ).

\subsection{Quantification of WLR}

With a gas/oil/water flow, capacitance can be measured from multiple electrodes surrounding a cross-section of a pipe, and the phase fraction of the fluid mixture of gas, oil and water can be potentially determined from the reconstructed permittivity distribution as the permittivities of the gas, oil and water are known to be $\sim 1.0, \sim 2.2$ and $\sim 80$, respectively (although the permittivities of oil and water are also a function of composition, pressure and temperature). ECT is suitable for measuring oil-continuous multiphase flows, because in this 
case water is dispersed in oil in the liquid phase and the liquid is non-conductive.

To calibrate the ECT system, raw capacitance measurements for low calibration, $C_{l}$, (28 for an eightelectrode system) are normally made by using a material with known low-permittivity $\left(\varepsilon_{l}\right)$ (such as an empty pipe with air or dry gas), followed by raw capacitance measurements for high calibration, $C_{h}$, by using a material of known high permittivity $\left(\varepsilon_{h}\right)$ (such as full-pipe oil or an oil-water uniform mixture with a known WLR).

In this work, the following series-capacitance normalization model (Yang and Byars 1999) is used to derive the normalized capacitance $C_{n}$ from the raw capacitance $C_{m}$ :

$$
C_{n}=\frac{1 / C_{m}-1 / C_{l}}{1 / C_{h}-1 / C_{l}}
$$

The permittivity of a homogeneous oil-continuous oil/water mixture depends on the WLR, which can be expressed by Ramu-Rao's model (Xie 2006):

$$
\varepsilon_{\text {liquid }}=\varepsilon_{\text {oil }} \frac{1+2 \mathrm{WLR}}{1-\mathrm{WLR}}
$$

where $\varepsilon_{\text {liquid }}$ is the permittivity of the oil/water mixture, and $\varepsilon_{\text {oil }}$ is the permittivity of oil.

Here the assumption is that the liquid in the sensing field of the measurement system is a homogeneous mixture of oil and water. If such an assumption is not true, then deriving the water fraction or WLR from the measured (apparent) permittivity using a homogeneous mixing model may result in inaccurate results.

For the gas-liquid annular multiphase flow, the permittivity of the gas-liquid mixture can be modelled as

$$
\varepsilon_{m}=\alpha_{\text {liquid }}^{x} \varepsilon_{\text {liquid }}+\left(1-\alpha_{\text {liquid }}^{x}\right) \varepsilon_{\text {gas }}
$$

where $\alpha_{\text {liquid }}$ is the liquid volume fraction (or holdup), $\varepsilon_{\text {gas }}$ is the permittivity of gas and $x$ is an empirical parameter, which is also measurement path dependent.

Equations (3) and (4) imply that the permittivity of the gas-liquid flow is related to the WLR and liquid holdup $\alpha_{\text {liquid. }}$ Using ECT with multi-view mixture-permittivity measurements, it is possible to measure these parameters (to be discussed).

To measure the oil/water mixture with various WLRs, the ECT sensor was calibrated using the oil/water mixture with $\mathrm{WLR}=0.35$. In this case, the measured normalized capacitance $C_{n}$ for full-pipe oil (WLR $=\sim 0, \mathrm{GVF}=0$ ) is 0.65 and increases almost linearly with the increase in WLR up to WLR $=\sim 0.35$. The nonlinearity effect of the pipewall capacitance can be excluded in the measured normalized capacitance $C_{n}$ as follows.

Let us assume that the effective capacitance of the insulating pipe wall $\left(8 \mathrm{~mm}\right.$ thick PEEK material) $\left(C_{w}\right)$ is in series with the fluid (unknown) capacitance $C_{x}$ and that $C_{w}$ is stable. The measured raw capacitance of the unknown fluid/pipe system $\left(C_{m}\right)$ and that of the low-permittivity calibration material $\left(C_{l}\right)$ and the high-permittivity calibration material $\left(C_{h}\right)$ are then

$$
\begin{aligned}
& 1 / C_{m}=1 / C_{w}+1 / C_{x}\left(\varepsilon_{m}\right) \\
& 1 / C_{l}=1 / C_{w}+1 / C_{x}\left(\varepsilon_{l}\right) \\
& 1 / C_{h}=1 / C_{w}+1 / C_{x}\left(\varepsilon_{h}\right) .
\end{aligned}
$$

The normalized capacitance $C_{n}$ according to equation (2) hence becomes

$$
\begin{aligned}
C_{n} & =\frac{\left(1 / C_{w}+1 / C_{x}\left(\varepsilon_{m}\right)\right)-\left(1 / C_{w}+1 / C_{x}\left(\varepsilon_{l}\right)\right)}{\left(1 / C_{w}+1 / C_{x}\left(\varepsilon_{h}\right)\right)-\left(1 / C_{w}+1 / C_{x}\left(\varepsilon_{l}\right)\right)} \\
& =\frac{1 / C_{x}\left(\varepsilon_{m}\right)-1 / C_{x}\left(\varepsilon_{l}\right)}{1 / C_{x}\left(\varepsilon_{h}\right)-1 / C_{x}\left(\varepsilon_{l}\right)} .
\end{aligned}
$$

In this way, the wall capacitance $C_{w}$ is removed in the measured normalized capacitance $C_{n}$, by the use of the serial-capacitance model.

The fluid only (unknown) capacitance $C_{x}$ can be assumed to be proportional to the dielectric constant $\varepsilon_{m}$ of the bulk fluid (where $k$ are proportional constants), namely

$$
C_{x}\left(\varepsilon_{m}\right)=k \varepsilon_{m}
$$

The low-calibration $\left(\varepsilon_{m}=\varepsilon_{l}\right)$ capacitance and high-calibration $\left(\varepsilon_{m}=\varepsilon_{h}\right)$ capacitance become

$$
\begin{aligned}
& C_{x}\left(\varepsilon_{l}\right)=k \varepsilon_{l} \\
& C_{x}\left(\varepsilon_{h}\right)=k \varepsilon_{h} .
\end{aligned}
$$

The measured normalized capacitance $C_{n}$ can then be related to the ultimately desired flow-mixture permittivity $\varepsilon_{m}$ :

$$
C_{n}=\frac{1 / C_{x}\left(\varepsilon_{m}\right)-1 / C_{x}\left(\varepsilon_{l}\right)}{1 / C_{x}\left(\varepsilon_{h}\right)-1 / C_{x}\left(\varepsilon_{l}\right)}=\frac{1 / \varepsilon_{m}-1 / \varepsilon_{l}}{1 / \varepsilon_{h}-1 / \varepsilon_{l}} .
$$

Rearranging equation (9) we have

$$
\varepsilon_{m}=\frac{1}{C_{n}\left(1 / \varepsilon_{h}-1 / \varepsilon_{l}\right)+1 / \varepsilon_{l}} .
$$

The purpose of the new inversion steps as described above is to achieve a physics-based quantification of WLR from the raw capacitance measurements by (1) converting the measured normalized capacitance $C_{n}$ to a corresponding mixture permittivity $\varepsilon_{m}$, and by (2) converting $\varepsilon_{m}$ to WLR (and gas/liquid fraction) for the oil-water (and gas-liquid) mixture, based on a dielectric mixing law(s). The outcome of the key step (1) also permits a more quantitative reconstruction of the mixture permittivity image based on the multi-view $\varepsilon_{m}$ data, for liquid-liquid and gas-liquid flows, rather than a conventional, qualitative grey- or colour-level image reconstruction based on the normalized capacitance $C_{n}$. The new quantitative image reconstruction method based on equation (10) is shown in figure 2. In this approach, the mixture permittivity $\varepsilon_{m}$ (rather than $C_{n}$ ) is used as the input to the modified LBP algorithm and/or the correspondingly modified iterative image reconstruction algorithms (Yang and Peng 2003) to overcome the distribution-dependent 'soft-field' effect. The output of the reconstruction of the mixture-permittivity distribution $\varepsilon_{m}(x, y)$ can be used as the (optional) input to WLR and/or gas-fraction interpretation.

\subsection{Model-based image reconstruction algorithm}

As mentioned before, in this research, a flow-conditioning device is used to form annular gas-liquid flows. A modelbased image reconstruction algorithm is used to quantitatively derive the WLR and the thickness of the liquid layer $(h)$ of the annular flows. The LBP algorithm is also used to generate images for the real-time monitoring purpose. Starting from an LBP image the initial $h$ and WLR can be obtained and the model-based algorithm further modifies the results iteratively. 


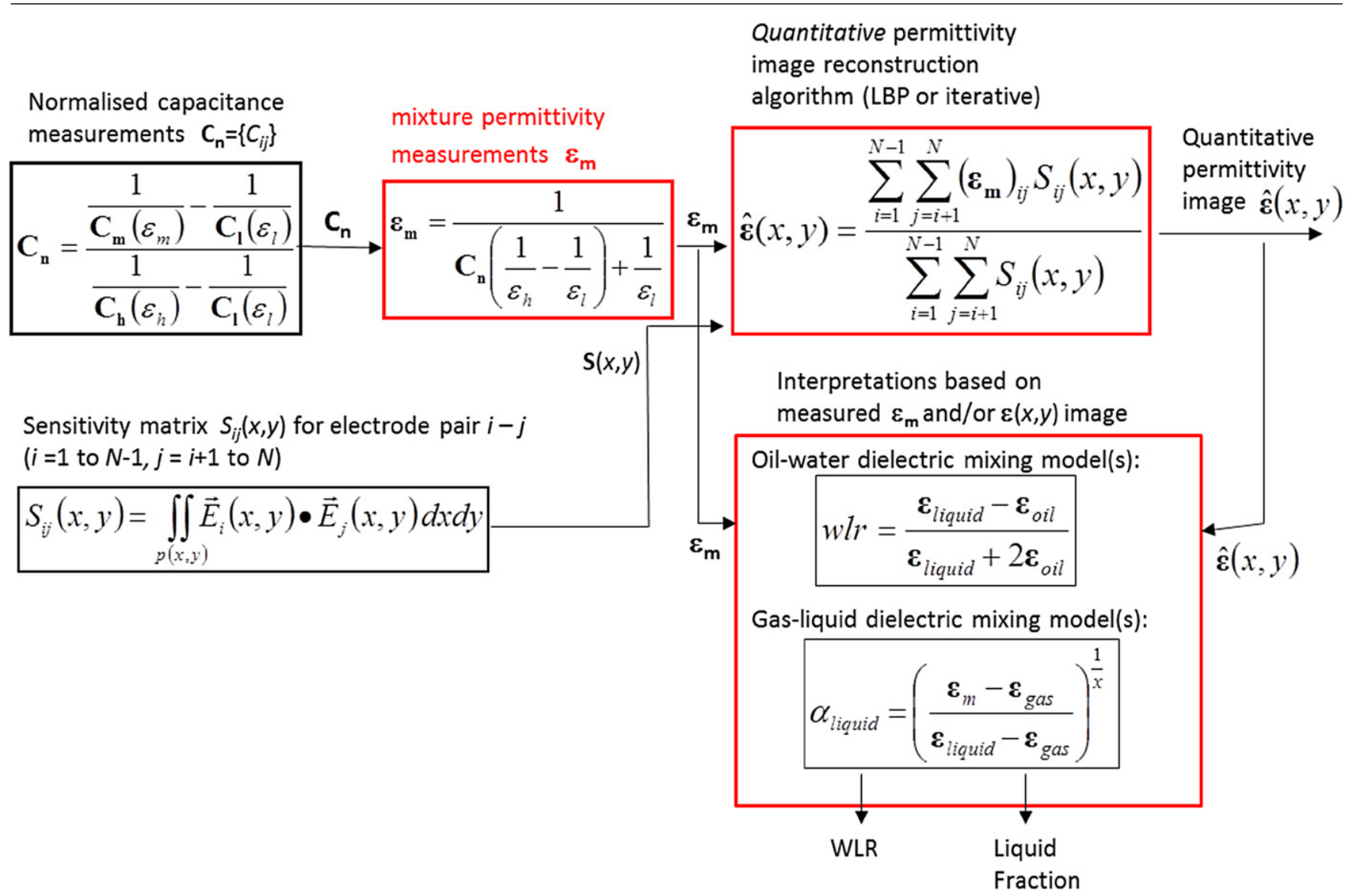

Figure 2. New inversion steps of permittivity image reconstruction and derivation of WLR and liquid fraction.

Figure 3(a) shows the eight-electrode ECT sensor for calculating capacitance from a given gas-liquid annular permittivity distribution based on the finite-element method (FEM). Figure 3(b) shows the block diagram of the modelbased image reconstruction algorithm based on previous research work (Isaksen and Nordtved 1993, Isaksen et al 1994). An optimization routine is used to minimize the discrepancy between the FEM simulated capacitance and the measured capacitance.

From an initial image obtained from LBP, the normalized capacitance $C_{n \text {,sim }}$ is calculated based on the serial-capacitance model given by equation (2) and compared with the corresponding measured normalized capacitance $C_{n \text {,mea }}$. The difference is used as the input to an optimization routine, to modify the distribution with the annular-flow parameters (i.e. WLR and $h$ ). The capacitance is re-calculated from the new distribution, and new $C_{n \text {,sim }}$ will again be compared with $C_{n \text {,mea }}$. The iteration process continues until an acceptable minimum difference between $C_{n \text {,sim }}$ and $C_{n \text {,mea }}$ is found.

The optimization routine is based on the LevenbergMarquardt algorithm (Isaksen and Nordtved 1993, Isaksen et al 1994):

$$
\begin{aligned}
& F(\beta)=\frac{1}{2} \sum_{i=1}^{N}\left(f_{i}(\beta)\right)^{2}=\frac{1}{2}\|f(\beta)\|_{2}^{2} \\
& f_{i}(\beta)=C_{i}^{\text {sim }}(\beta)-C_{i}^{\text {mea }}
\end{aligned}
$$

where $N$ is the number of capacitance measurements; the $\beta$ variables include the flow parameters to be determined (i.e.
WLR and $h$ ). Because the number of flow parameters is less than the number of capacitance measurements, it is possible to obtain a determined solution.

In the case of an axis-symmetrical annular flow distribution, only four independent capacitance-measurement groups (Wang et al 2009, Yang et al 2004) can be obtained, i.e. from adjacent pair, one-electrode apart, two-electrode apart and opposite pair. Note that the number of capacitancemeasurement groups is sufficient to determine the two unknowns. In this research, the axis-symmetrical gas core is largely generated in horizontal swirl flows, where the centrifugal force is much greater than gravity and hence the eccentricity of the gas core can be ignored.

\section{Initial test in Schlumberger}

The designed measurement system was initially tested on a flow loop in Schlumberger Gould Research to evaluate the performance of the ECT sensor. The ECT sensor was mounted on a horizontal Perspex pipe with ID $=50 \mathrm{~mm}$ and $\mathrm{OD}=$ $60 \mathrm{~mm}$. Data were collected from $60 \mathrm{~s}$ running for each flow condition. The mixture fluid with low-viscosity $(\sim 2 \mathrm{cP})$ kerosene $\left(\varepsilon_{\text {oil }} \approx 2.2\right)$, local tap water $\left(\varepsilon_{\text {water }} \approx 79\right)$ and nitrogen gas was used. The flow mixture of gas, oil and water in the test section passed horizontally through the sensor section as shown in figure 4.

The tested GVF range was from $0 \%$ to $\sim 80 \%$ with line pressure up to about 2 bar. The total liquid flow rate $Q_{l}$ was 


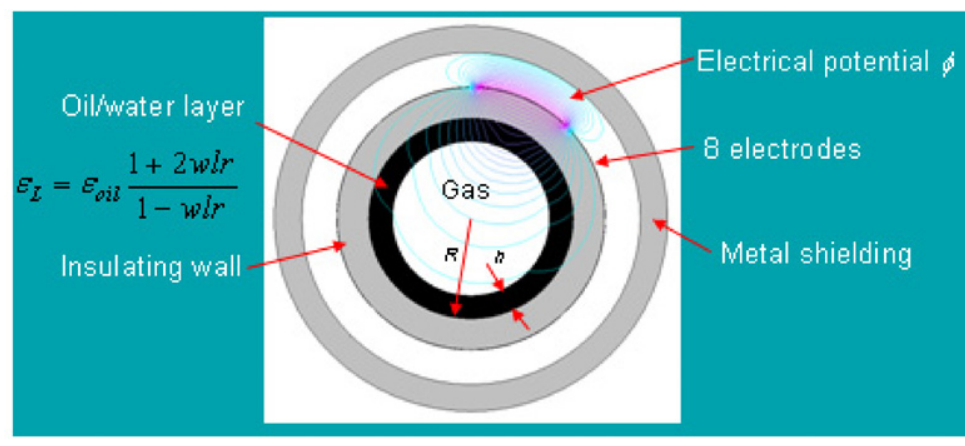

(a) Annular gas-liquid distribution

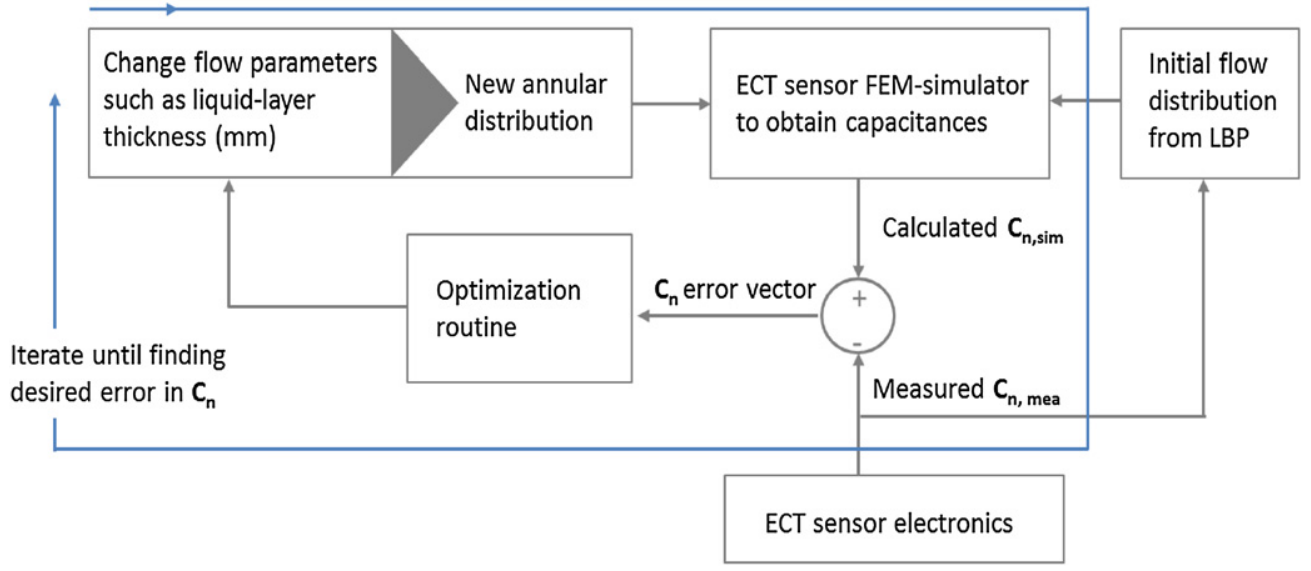

(b) Model-based image reconstruction algorithm

Figure 3. ECT sensor model and model-based iterative algorithm..

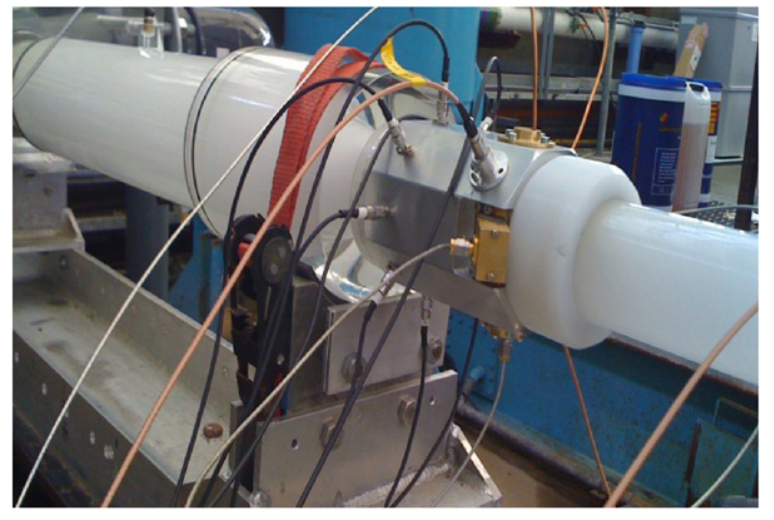

Figure 4. ECT sensor on Schlumberger's flow-loop.

10-30 $\mathrm{m}^{3} \mathrm{~h}^{-1}$. For a horizontal flow, the flow-conditioning device is located upstream of the Venturi section to form a largely axis-symmetrical gas-liquid annular flow. Figure 5 shows the time-average normalized capacitance measurements $C_{n}$ for the gas/oil/water oil-continuous flows. The ECT sensor was calibrated online with the oil/water mixture with WLR = $30 \%$ (at $\mathrm{GVF}=0$, and a high liquid rate of $Q_{l}=30 \mathrm{~m}^{3} \mathrm{~h}^{-1}$ to ensure good mixing) and full-pipe air with $\varepsilon=1.0$.

The reasons behind selecting the high calibration point with WLR $=30 \%$ were that (1) it was difficult to mix kerosene and water very well when the WLR increased beyond 30\% (note that the phase-inversion WLR for the lowviscosity kerosene and water mixture is around 35\%), and (2) the capacitance measuring electronics would be short- circuited due to the high conductivity of the mixture fluid. As indicated in figure 5(a), when the gas/oil/water flow had a low liquid flow rate (i.e. $Q_{l}=10 \mathrm{~m}^{3} \mathrm{~h}^{-1}$ ) and a high WLR (i.e. WLR $\geqslant 30 \%$ ), some capacitance measurements presented negative values, indicating that oil and water were not well mixed and a water-rich layer existed in a near pipe wall region. With the increase in liquid flow rate, i.e. $Q_{l}=$ 20 and $30 \mathrm{~m}^{3} \mathrm{~h}^{-1}, \mathrm{GVF}=0$, the flow velocity increases, the mixture of the oil/water oil-continuous is more uniform and accurate capacitance measurements can be obtained.

Figure 5 also indicates that with the increase in the WLR, the measured capacitance increases for flows with the same GVF. With the increase in GVF, the measured capacitance decreases for flows with a fixed WLR. For a flow with a lower GVF (e.g. $\leqslant 0.25$ ), the 'one-electrode apart' capacitance is mainly sensitive to the WLR, because such a flow has a thick liquid layer, the sensing region of the 'one-electrode apart' electrode pairs mainly cover the near-wall region, hence the effect of the gas core reduces. However, both WLR and GVF affect the 'opposite-pair electrode' cross-pipe measurements.

Figures 6(a) and $(b)$ show the calculated thickness $(h)$ and the WLR of the liquid layer of the gas-liquid oil-continuous flows by the model-based iterative algorithm. With the increase in GVF, the estimated $h$ decreases, see figure 6(a). Note that there was a varying-size gas core when $\mathrm{GVF}=0$ due to the trapped gas in the flow loop. Since there is no reliable reference to calibrate the thickness measurement, the accuracy of this measurement cannot be defined for the test. The deviation between the estimated WLR and the reference WLR is less 


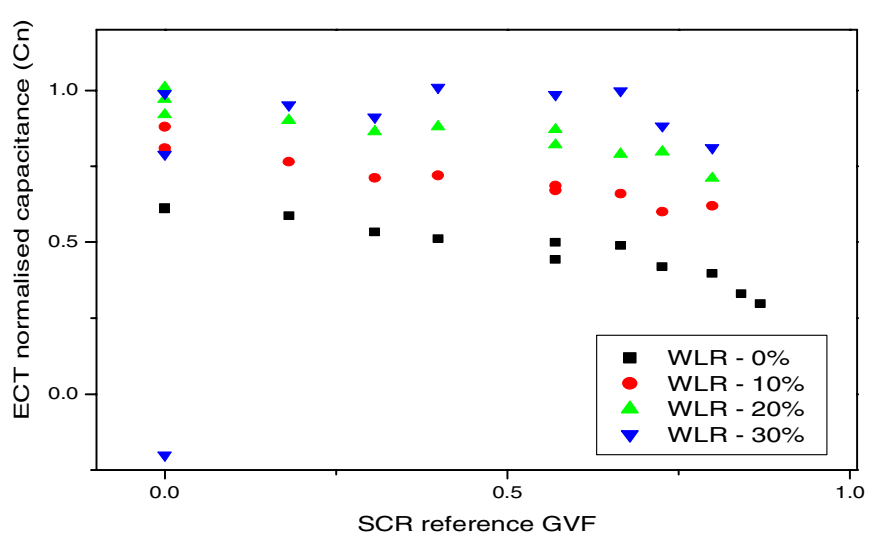

(a) 1-electrode apart $\mathbf{C}_{\mathbf{n}}$

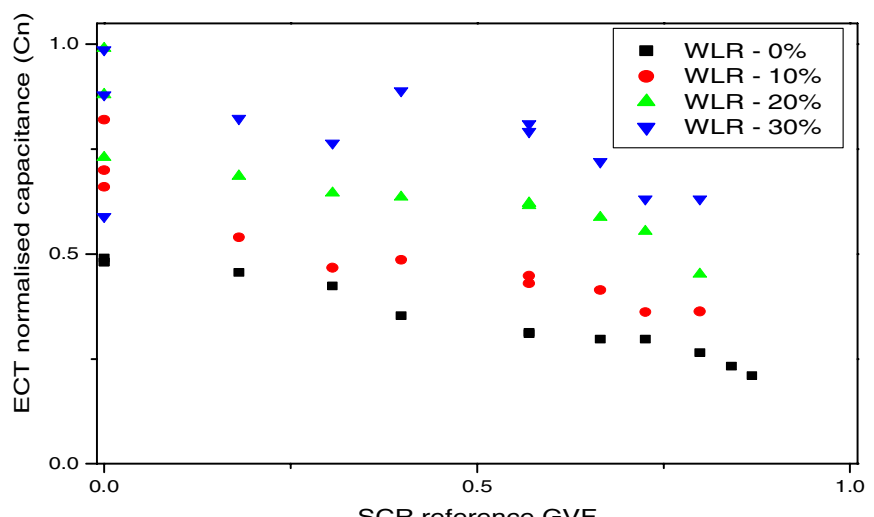

(b) 2-electrode apart $\mathbf{C}_{\mathbf{n}}$

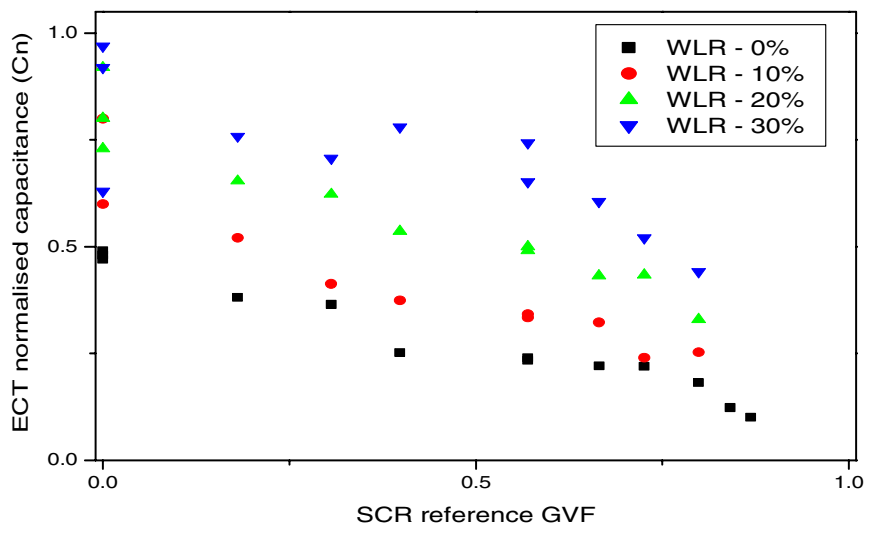

(c) Opposite pair electrode $\mathbf{C}_{\mathbf{n}}$

Figure 5. Normalized capacitance based on $60 \mathrm{~s}$ time-average results.

than $\pm 5 \%$ absolute. As shown in figure $6(b)$, when the flow has a high WLR (i.e. 30\%) and low liquid flow rate (i.e. $Q_{l}=10 \mathrm{~m}^{3} \mathrm{~h}^{-1}$ ), the error of the estimated WLR is more than $5 \%$. This corresponds to the intermittently oil- and watercontinuous, non-uniform oil-water flows.

\section{Test in TUV-NEL}

\subsection{Test facilities}

A redesigned eight-electrode ECT sensor (see figure 1) was tested as part of the experimental apparatus on TUV-NEL's Multiphase Flow Facility with crude oil and salty water.

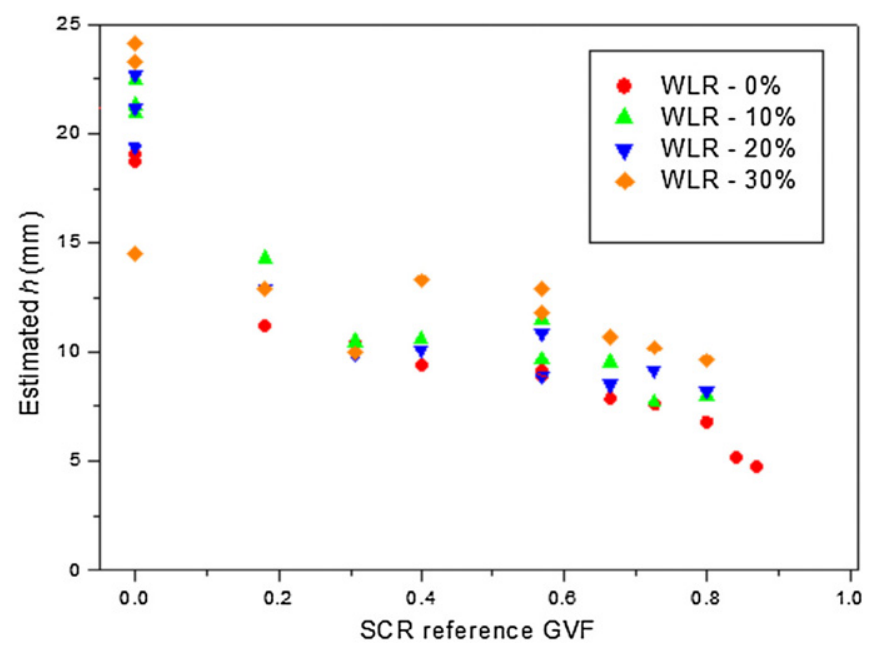

(a) Measured h (mm)

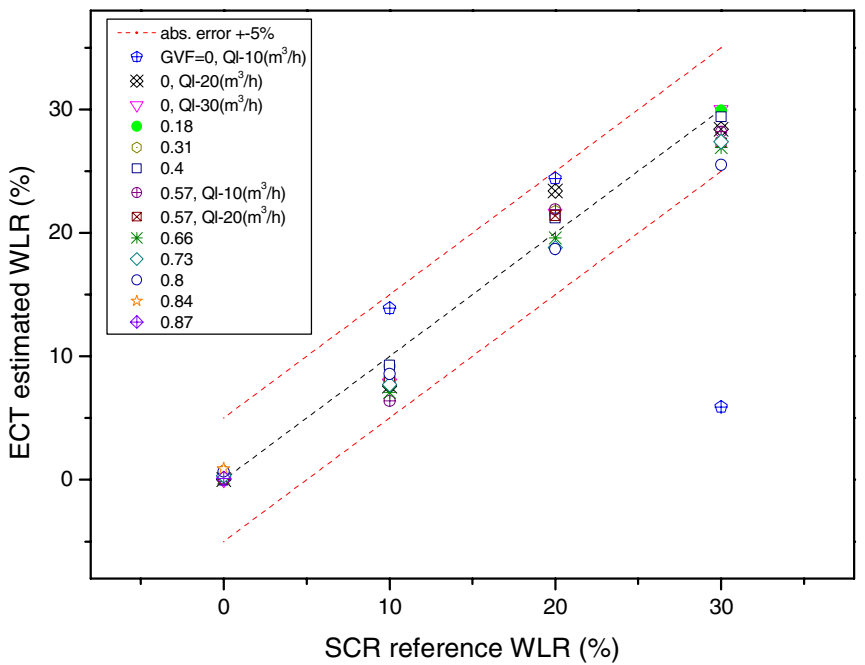

(b) Measured WLR (\%)

Figure 6. Time-average ECT measured results from the modelbased algorithm.

Figure 7(a) shows the facility consisting of a three-phase separator, which contains working bulk fluids, and a test loop section. Oil is supplied from the separator to the main oil pump and then to the reference oil flow metering section. Oil and water are re-circulated around the test facility using two variable speed pumps. The sampling loops provide information on any cross contamination in the oil and water process streams. Heat exchangers are used to stabilize the temperature of the working fluid, $\sim 40{ }^{\circ} \mathrm{C}$.

The mixture fluid with crude oil (i.e. Forties/Oseberg crude and Exxon D80) with $\varepsilon_{\text {oil }}=2.2$ and water with $\varepsilon_{\text {water }} \approx$ 75 passes through the test section. The viscosity of crude oil at $40{ }^{\circ} \mathrm{C}$ is $\sim 9 \mathrm{cP}$. The salinity of water for the test is $\sim 50 \mathrm{~g} \mathrm{~L}^{-1}\left(\mathrm{MgSO}_{4}\right)$. Nitrogen is used as the gas phase and can be delivered up to $0.3 \mathrm{~kg} \mathrm{~s}^{-1}$ by evaporation of fluid nitrogen on demand (Ross et al 2010). The mixture fluid of gas, oil and water in the test section runs horizontally to the ECT sensor (see figures $7(b)$ and $(c)$ ). GVF ranges from $0 \%$ to $\sim 95 \%$ with a total liquid flow rate of $10-40 \mathrm{~m}^{3} \mathrm{~h}^{-1}$. For horizontal pipe flows, the flow-conditioning device is located 


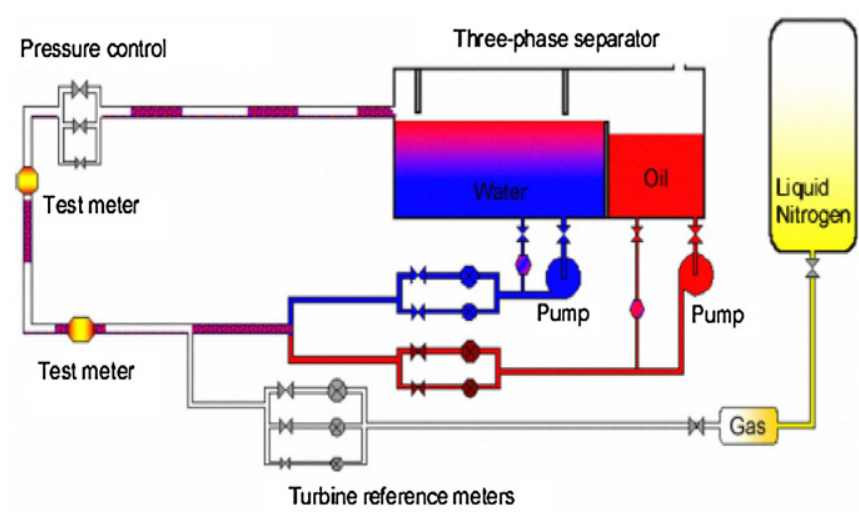

(a) TUV-NELs M ultiphase flow Facility

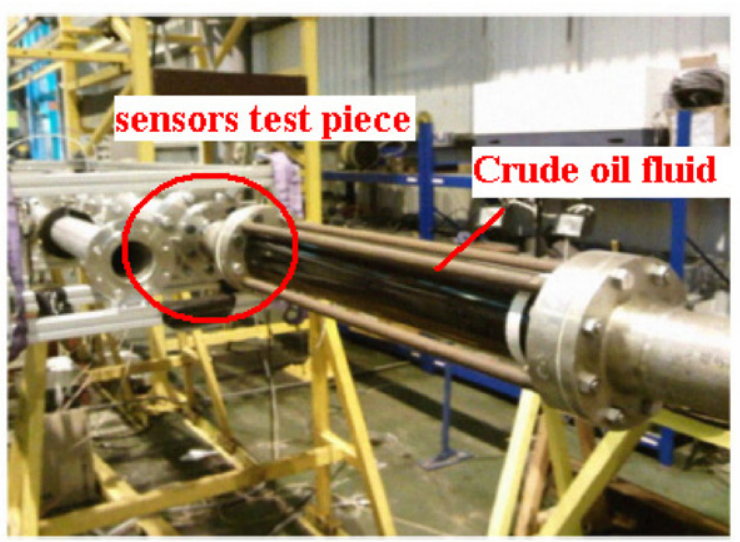

(b) Sensor spool-piece

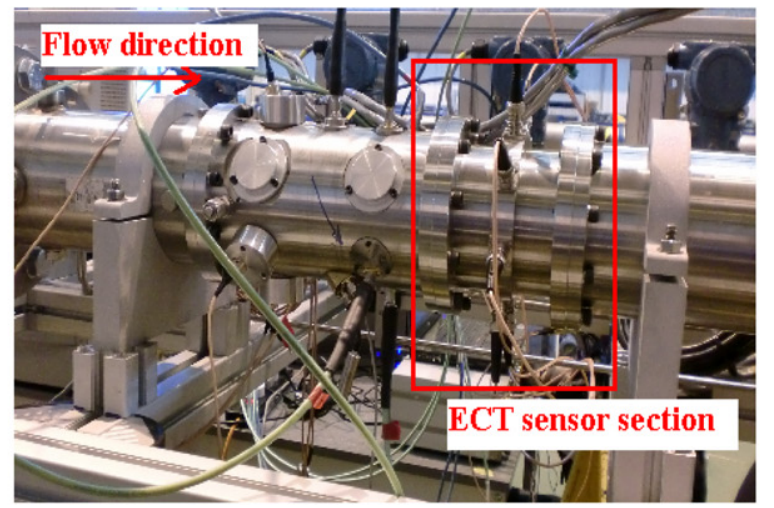

(c) ECT sensor section

Figure 7. ECT sensor on TUV-NEL's Multiphase Flow Facility.

upstream of the Venturi section, to generate gas-liquid flows with an axis-symmetrical gas core. The ECT sensor section is located at the Venturi throat section with the inner radius of about $26 \mathrm{~mm}$. The inlet line pressure is up to $7 \mathrm{bar}$.

\subsection{Measurement of oil/water oil-continuous flows}

In this case, $\mathrm{GVF}=0$ and well-mixed oil/water mixture with varying WLR was measured. Three different flow conditions were tested: horizontal swirl flows with the flow conditioning device, horizontal non-swirl flows without the flow conditioning device and vertical non-swirl flows. The phase-inversion transition point between oil-continuous and water-continuous flows can be at WLR $=50-60 \%$.

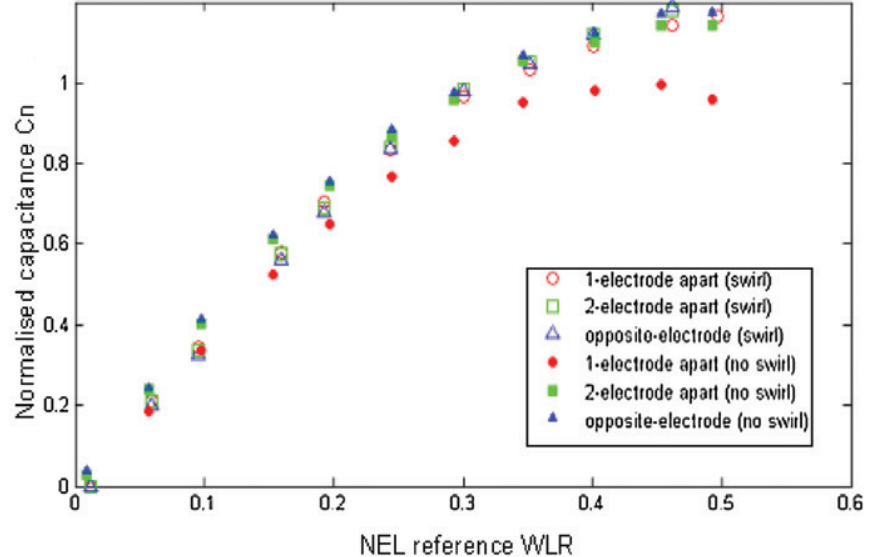

(a) $C_{n}$ against WLR

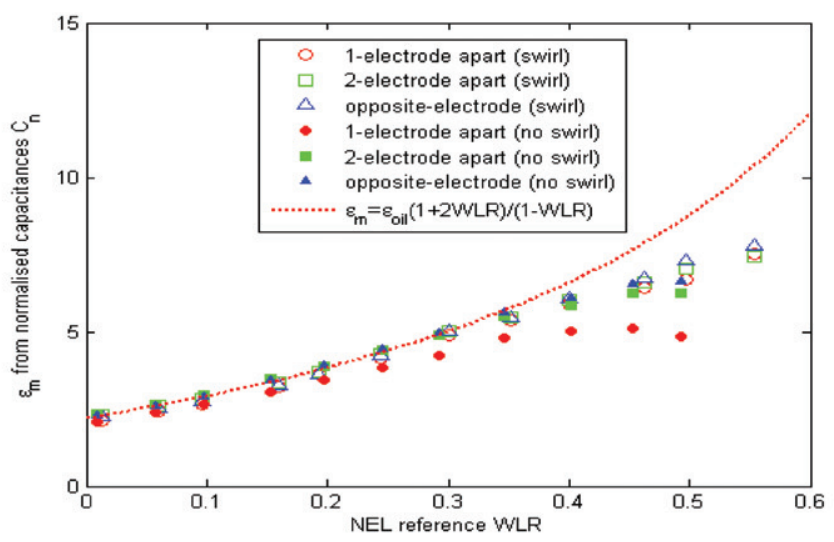

(b) Estimated $\varepsilon_{m}$ from $C_{n}$

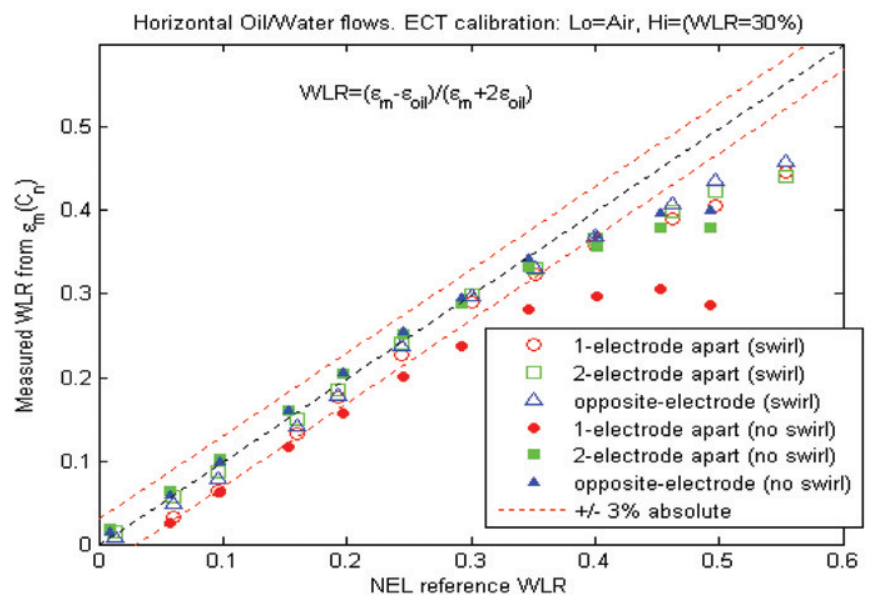

(c) Estimated WLR from $\varepsilon_{m}$

Figure 8. ECT results of horizontal swirl and non-swirl oil/water oil-continuous flows, total liquid flow rate $Q_{l}=40 \mathrm{~m}^{3} \mathrm{~h}^{-1}$.

The capacitance electrodes can be short-circuited by watercontinuous liquid if WLR $\geqslant 50 \%$ with a sufficiently high bulk liquid conductivity. It was also observed that the response of the capacitance sensors became increasingly non-linear as WLR increased beyond 35\%, see figure 8(a). Therefore, in this work, for calculating normalized capacitance, an oil/water (well-mixed) flow with WLR $=30$ or $35 \%$ was used for high calibration and an oil flow (with WLR $0 \%$ ) for low calibration. 
In figure $8(a)$ we can see that for the three different electrode-pair groups ('one-electrode apart', 'one-electrode apart', 'opposite-electrode'), and for both swirl and nonswirl horizontal flows at a relatively high total flow rate of $\sim 40 \mathrm{~m}^{3} \mathrm{~h}^{-1}$ (to ensure good mixing between oil and water), the time-average (over $\sim 60 \mathrm{~s}$ ) normalized capacitance $C_{n}$ increases almost linearly with the increase in WLR up to WLR $=\sim 0.35$.

Figure $8(b)$ gives the corresponding oil-water mixture permittivity $\left(\varepsilon_{m}\right)$ (plotted against the reference WLR) derived using equation (10) from the measured normalized capacitance (see figure $8(a)$ ). Excellent agreement with $\varepsilon_{m}$ of the homogeneous oil/water dielectric mixing model (equation (3)) can be observed for the WLR up to $35 \%$. From figure $8(b)$, we can see a small difference in the derived mixture permittivity $\left(\varepsilon_{m}\right)$ between the swirl and non-swirl (oilcontinuous) horizontal flows with WLR up to $35 \%$, except that there is a marked overall underestimate in $\varepsilon_{m}$ derived from the 'one-electrode-apart' (near-wall region) measurements for horizontal non-swirl flows, probably due to a non-ideal inline empty-pipe low-calibration where there could be a thin layer of liquid at the pipe underside. For other two groups of crosspipe measurements (i.e. 'two-electrode-apart' and 'oppositeelectrode'), the derived WLR from $\varepsilon_{m}$ is within $\pm 3 \%$ for WLR $<35 \%$ (see figure $8(c)$ ).

For the vertical oil-water non-swirl flows, because of the axi-symmetry and homogeneity of the vertical upward flow, we can hardly see any difference in the permittivity of mixture derived by ECT $\left(\varepsilon_{m}\right)$ with WLR up to $35 \%$, among the 'one-electrode-apart' (near-wall region) measurements, the near cross-pipe measurements ('two-electrode-apart') and the cross-pipe measurements ('opposite-electrode'). The WLR derived by ECT from the $\varepsilon_{m}$ data is within $\pm 3 \%$ absolute, for WLR $<35 \%$.

Since the cross-sectional distribution of the vertical upward oil-water (non-swirl) flows, at a relatively high flow rate of $\sim 40 \mathrm{~m}^{3} \mathrm{~h}^{-1}$, is considered to be axis-symmetrical and homogeneous, and since there is hardly any difference in the permittivity of mixture derived by ECT (WLR $\leqslant$ $35 \%$ ), between the vertical non-swirl and horizontal swirl oilwater flows, for the 'one-electrode-apart' (near-wall region) measurements (see figure $9(a)$ ), the 'two-electrode-apart' near cross-pipe measurements (see figure $9(b)$ ) and the 'oppositeelectrode' cross-pipe measurements (see figure 9(c)), the oilwater distribution of horizontal (oil-continuous) swirl flow is deduced to be homogeneous although the spatial average of measurements around the pipe circumference can remove some of the inhomogeneity in the permittivity distribution. This provides independent evidence that there is virtually no oil-water separation in the presence of a weak swirl that is damped by the high-viscosity liquid.

\subsection{Measurement of gas/oil/water oil-continuous flows}

In this section, the WLR and liquid holdup of horizontal swirl gas-liquid oil-continuous flows are estimated by ECT, based on the measured data from the near-wall, near crosspipe (or cross-diameter) and cross-pipe electrode pairs.

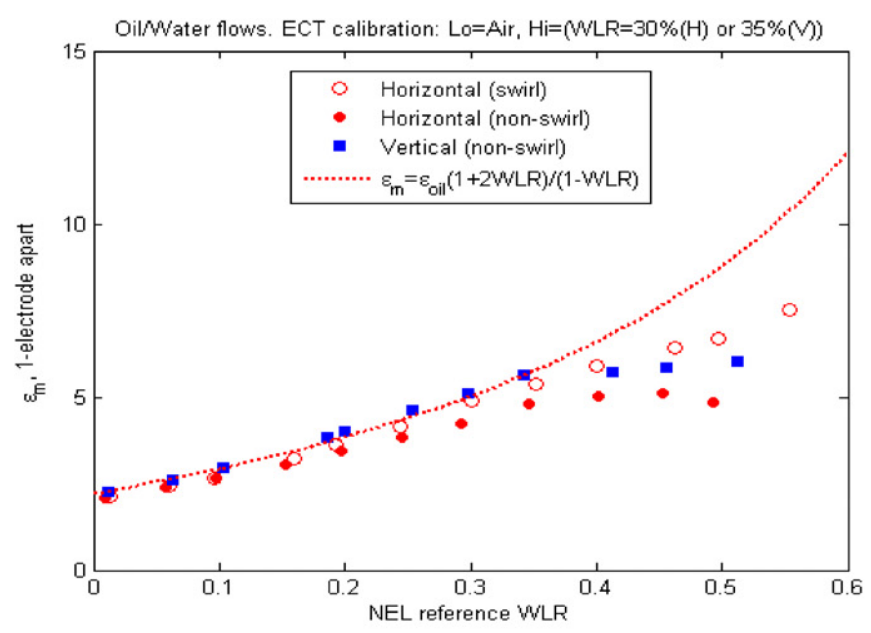

(a) 1-electrode apart

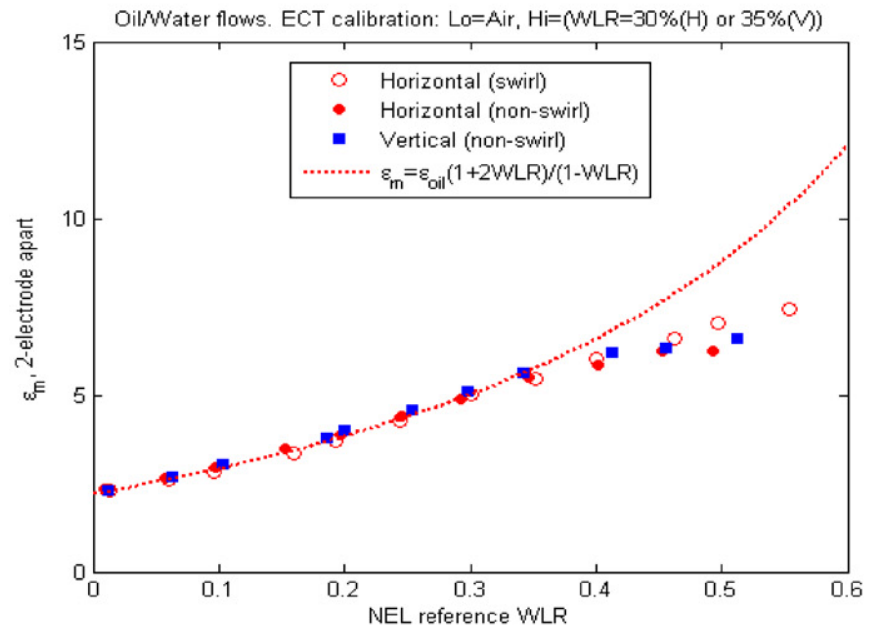

(b) 2-electrode apart

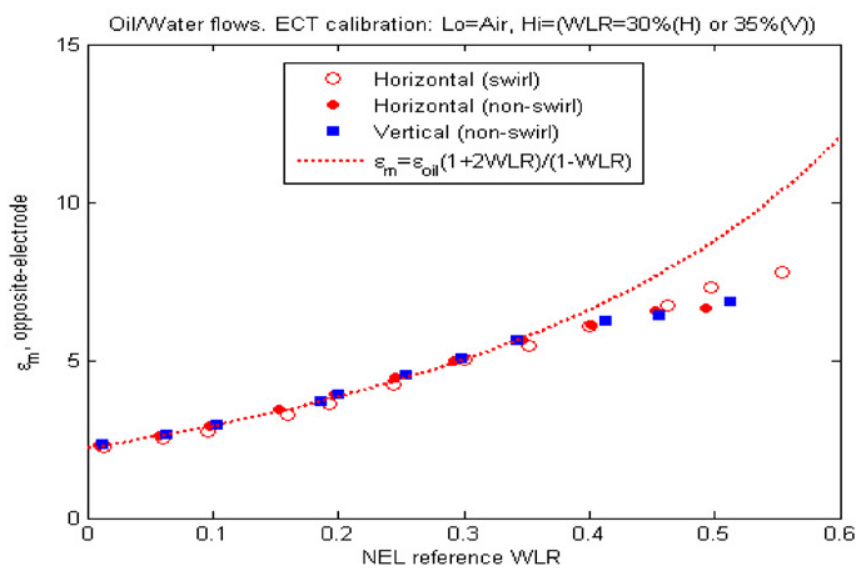

(c) opposite-electrode

Figure 9. Data for horizontal swirl, non-swirl and vertical non-swirl oil-water oil-continuous flows, the derived mixture permittivity $\varepsilon_{\mathrm{m}}$ from the normalized capactitance by equation (10) measured from the ECT electrode pairs. Low calibration: air; high calibration: $\mathrm{WLR}=30 \%$ for horizontal flows and WLR $=35 \%$ (rescaled) for vertical flows.

From the measured time-average normalized capacitance $C_{n}$ of gas-liquid swirl flows (see figure 10), the timeaverage permittivity of mixture can be determined (see figure 11) using equation (10), which can be applied to 

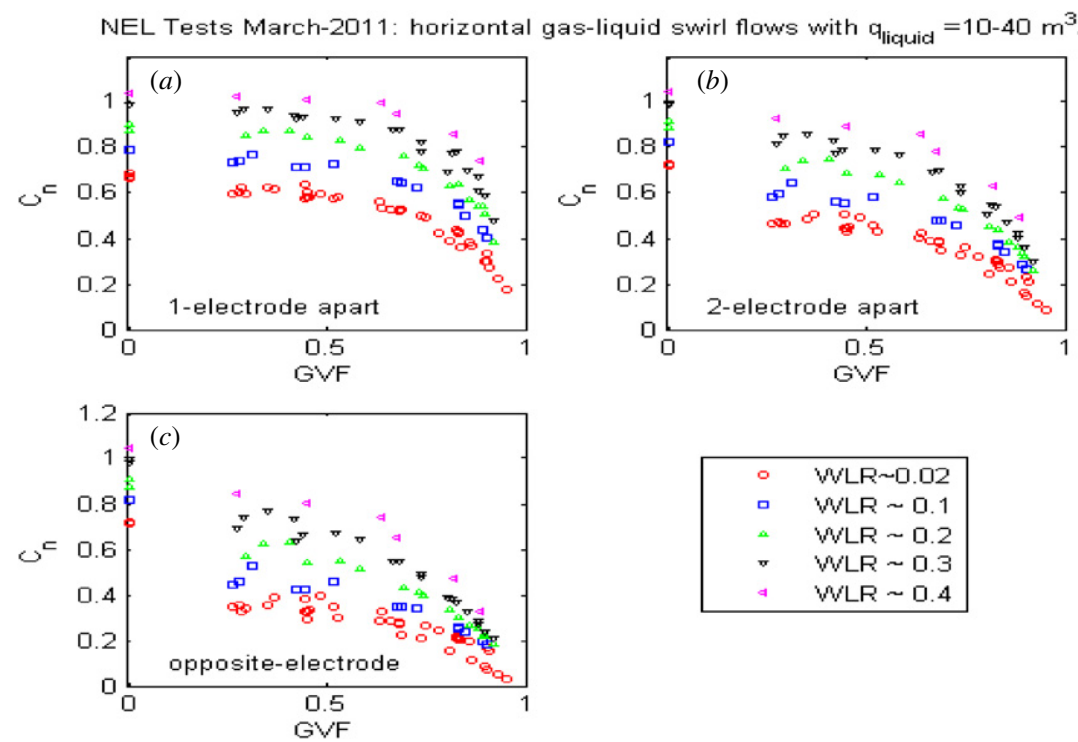

Figure 10. Plotted versus GVF of gas-liquid horizontal swirl flows with WLR up to $40 \%$, with time-average ( $\sim 60$ s) normalized capacitance $C_{n}$ for $(a)$ one-electrode apart, $(b)$ two-electrode-apart and $(c)$ opposite-electrode. Note that some $C_{n}$ of 'one-electrode apart' are close to saturation for WLR $\sim 40 \%$.
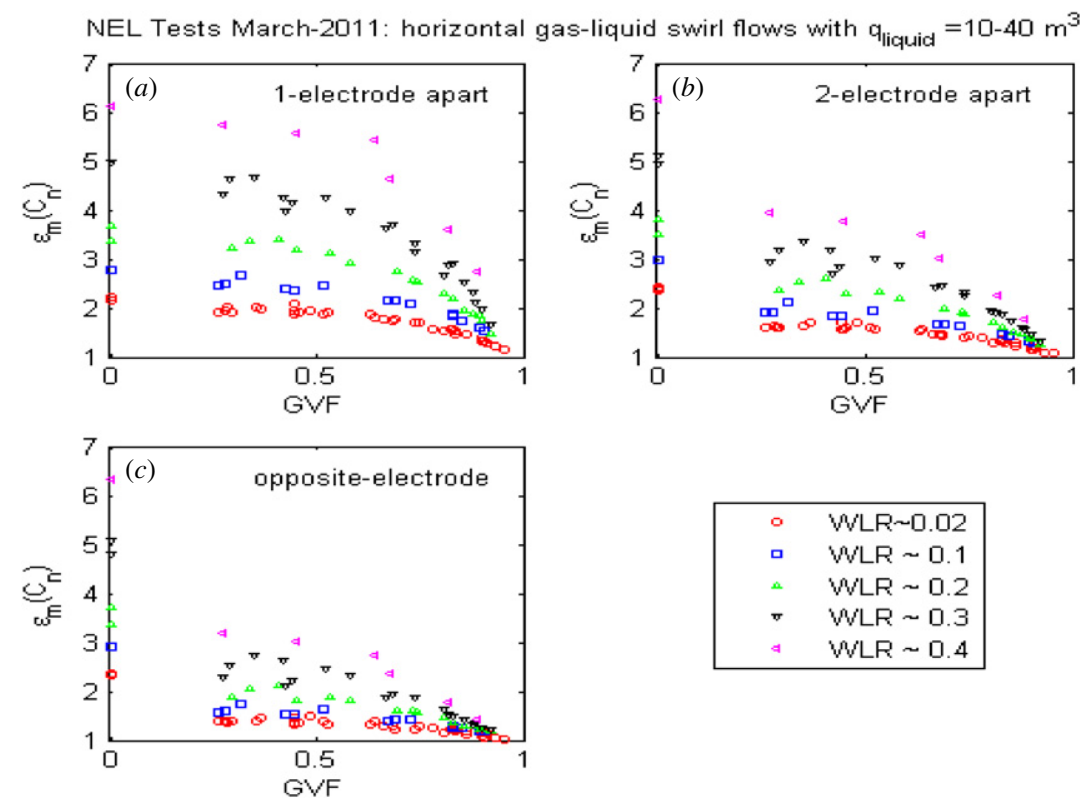

Figure 11. Plotted versus GVF of gas-liquid horizontal swirl flows with WLR up to $\sim 40 \%$, with permittivity of mixture $\varepsilon_{m}$ calculated from normalized capacitance $C_{n}$ in figure 10, for $(a)$ one-electrode apart, $(b)$ two-electrode apart and $(c)$ opposite-electrode. Note some $\varepsilon_{m}$ of 'one-electrode apart' are close to saturation for WLR $>\sim 40 \%$.

the instantaneous normalized capacitance measured every $10 \mathrm{~ms}$. Following the similar steps in figure 2, converting the measured instantaneous normalized capacitance $C_{n}(t)$ to the instantaneous permittivities of mixture $\varepsilon_{m}(t)$ permits a quantitative reconstruction of the instantaneous permittivity image $\varepsilon(r, t)$ based on $\varepsilon_{m}(t)$ for dynamic gas-liquid flows (see figure 12). Working in the mixture-permittivity parameter domain also permits the use of appropriate dielectric mixing models (equations (3) and (4)) for the determination of the WLR and/or liquid holdup of gas-liquid flows.

The purpose of using an inline swirl generator (i.e. a flowconditioning device) upstream of the Venturi inlet is to create a gas core and a liquid annulus at the throat measurement section. As can be seen from the instantaneous permittivity images illustrated in figure 12, a more steady gas core (or a smoother liquid layer) is formed at a relatively low $\operatorname{GVF}(\sim 25 \%)$ than at a relatively high GVF $(\sim 63 \%)$. As expected, the permittivity of oil/water mixture near the wall region increases when WLR increases. The real-time images reconstructed by the LBP are also useful to indicate gas-liquid flow regimes. Furthermore, it can be seen from figure 11 that the near-wall measurement ('one-electrode apart') at low GVFs preferentially measures the liquid-layer mixture permittivity, especially during the time intervals with the liquid-rich slugs passing (see figure 12, $\mathrm{GVF} \leqslant 0.4)$ 
GVF
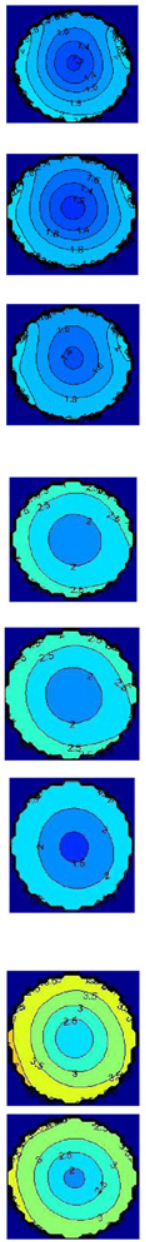
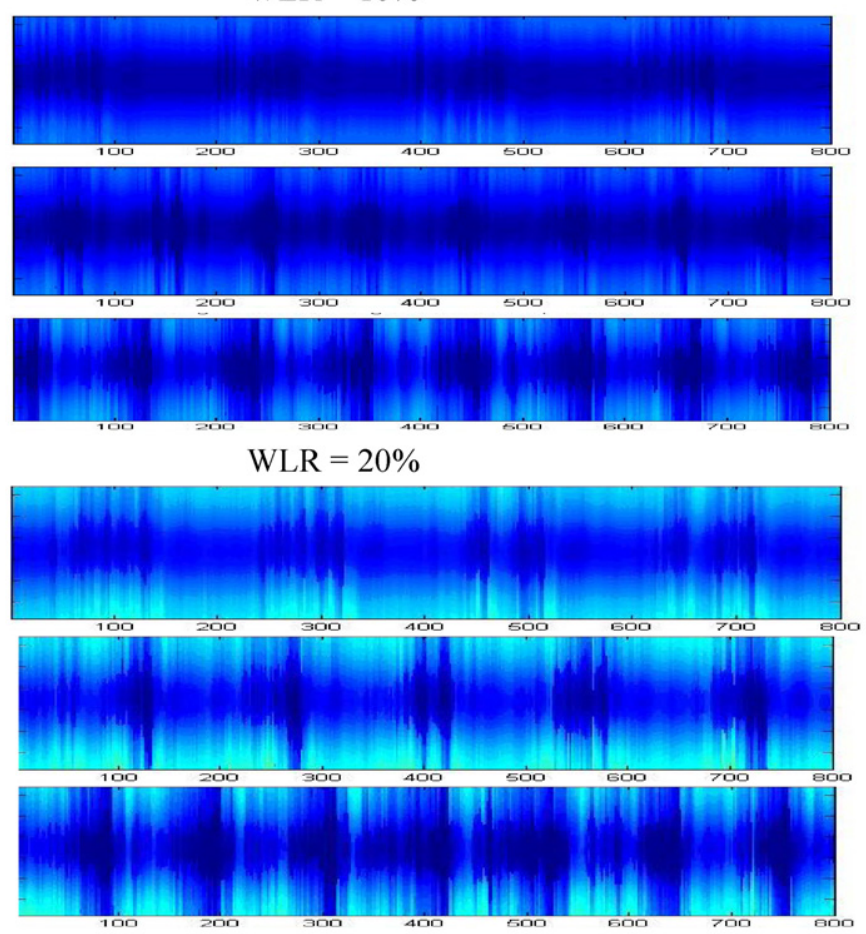

$$
\mathrm{WLR}=30 \%
$$
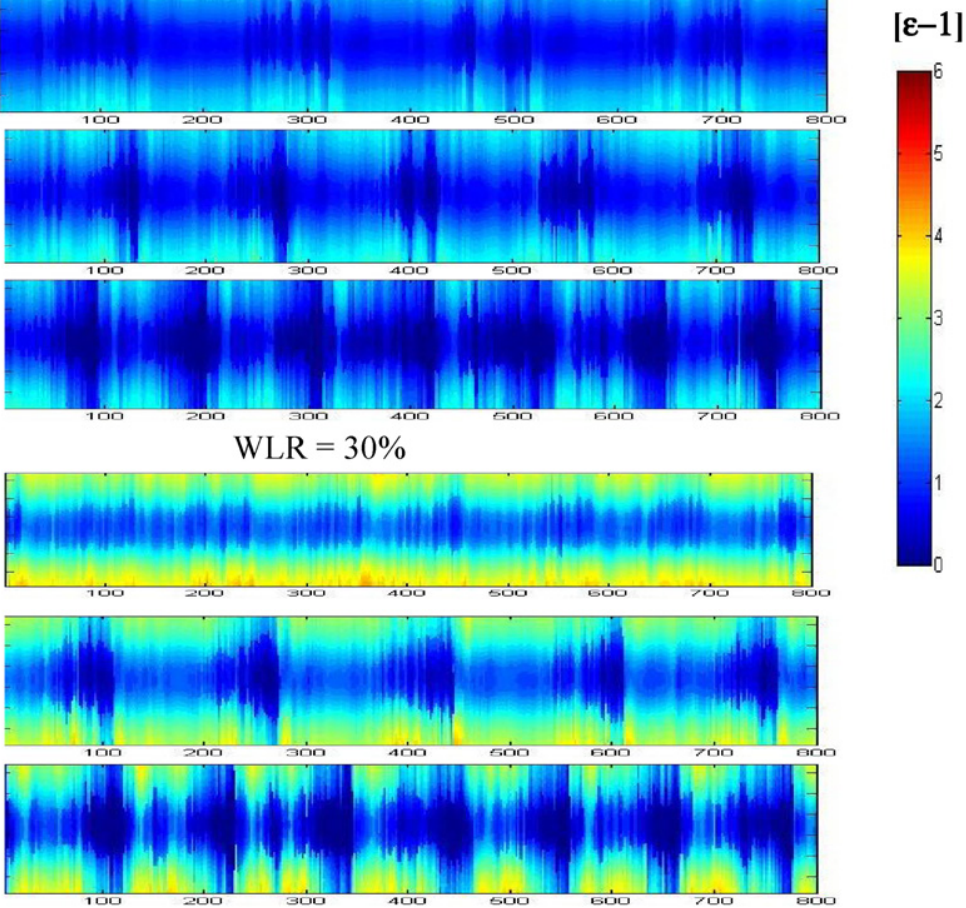

Figure 12. Left column: time-average (over $\sim 60 \mathrm{~s}$ ) cross-sectional images (permittivity-change colour scale $\varepsilon-1=0$ to 6 as shown on the far right) reconstructed based on the instantaneous permittivity of the gas-liquid mixture for horizontal gas-liquid swirl flows with the total liquid rate $Q_{l}=30 \mathrm{~m}^{3} \mathrm{~h}^{-1}$, WLR $=10 \%, 20 \%$ and $30 \%$, and $\mathrm{GVF}=25 \%, 40 \%$ and $63 \%$. Right column: corresponding to the left column, instantaneous longitudinal images stacked with over $8 \mathrm{~s}$ (imaging frame rate at 100 frames s${ }^{-1}$ ).

Assuming that the WLR of oil/water mixture is much more stable than the liquid holdup over a short measurement interval (say $\Delta T=10 \mathrm{~s}$, with one or more liquid slugs passing the sensors), this feature of near-wall localized sensing (in space) and/or dynamic liquid-slug mixture permittivity data capturing (in time, at a fast rate say $\Delta t=10 \mathrm{~ms}$ ) has the potential to yield a rolling estimate (within each $\Delta T$ interval) of a short time-average permittivity of liquid $\varepsilon_{\text {liquid }}(\Delta T)$.

This results in a rolling estimate of the liquid WLR by using an oil/water (uniform) mixing model such as RamuRao's equation (3):

$$
\mathrm{WLR}_{(\Delta T)}=\frac{\varepsilon_{\text {liquid }(\Delta T)}-\varepsilon_{\text {oil }}}{\varepsilon_{\text {liquid }(\Delta T)}+2 \varepsilon_{\text {oil }}} .
$$

From figure 13 , it can be seen that by capturing and analysing the permittivity of near-wall liquid-slug mixture, the WLR can be estimated to be $\pm 5 \%$ for GVF up to about $90 \%$, WLR up to $\sim 40 \%$ (largely within the permittivity linearity range of near-wall electrode pairs).
Regarding the estimate of the liquid holdup, figure 11 clearly indicates that, at the same WLR and GVF $(>0)$, the permittivity of mixture from cross-pipe (i.e. oppositeelectrode) measurement is much lower than that of the nearwall one, because of its full interrogation of the gas core. From the cross-pipe measurement of the instantaneous permittivity of the gas-liquid mixture, an instantaneous and time-average liquid holdup can be obtained by using a (non-uniform) gasliquid mixing model such as equation (4), with near-wall sensed liquid permittivity as the input, namely

$$
\begin{aligned}
\alpha_{\text {liquid }}(t) & =\left(\frac{\varepsilon_{m}(t)-\varepsilon_{\text {gas }}}{\varepsilon_{\text {liquid }(\Delta T)}-\varepsilon_{\text {gas }}}\right)^{\frac{1}{x}} \\
& \approx\left(\frac{\varepsilon_{m(\text { cross-pipe })}(t)-\varepsilon_{\text {gas }}}{\varepsilon_{m(\text { near-wall,liquid-slug })(\Delta T)}-\varepsilon_{\text {gas }}}\right)^{\frac{1}{x}} \\
\alpha_{\text {liquid }(\Delta T)} & =\left\langle\alpha_{\text {liquid }}(t \in \Delta T)\right\rangle
\end{aligned}
$$




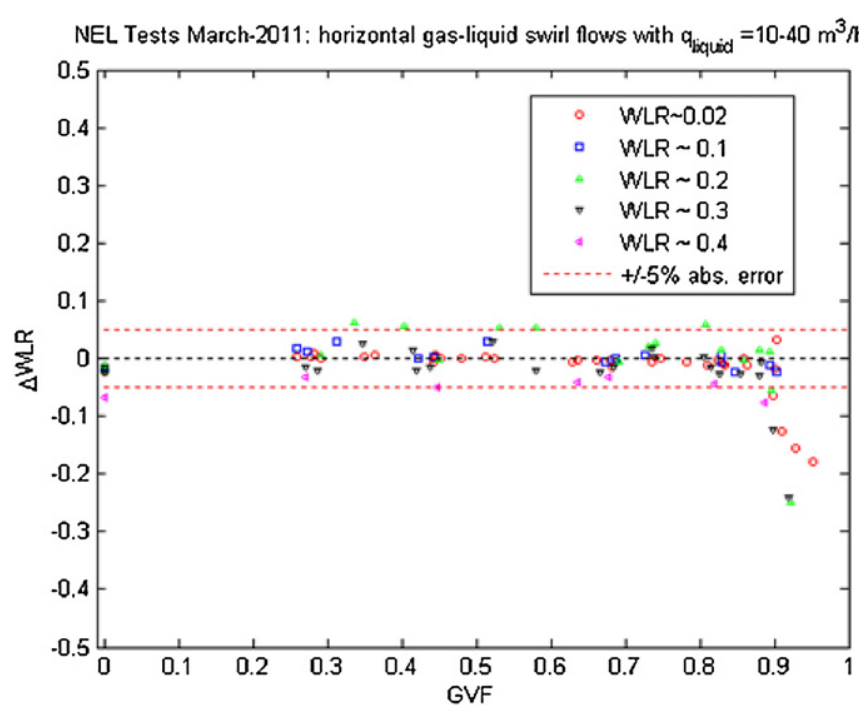

Figure 13. Plotted versus GVF, the absolute error in WLR estimated by ECT from the permittivity of the near-wall liquid-slug mixture by using equation (12). Swirl flow with WLR up to $\sim 40 \%(\sim 60 \mathrm{~s}$ data acquisition interval).

NEL Tests March-2011: horizontal gas-liquid swirl flows with $q_{\text {liquid }}=10-40 \mathrm{~m}^{3} / \mathrm{h}$

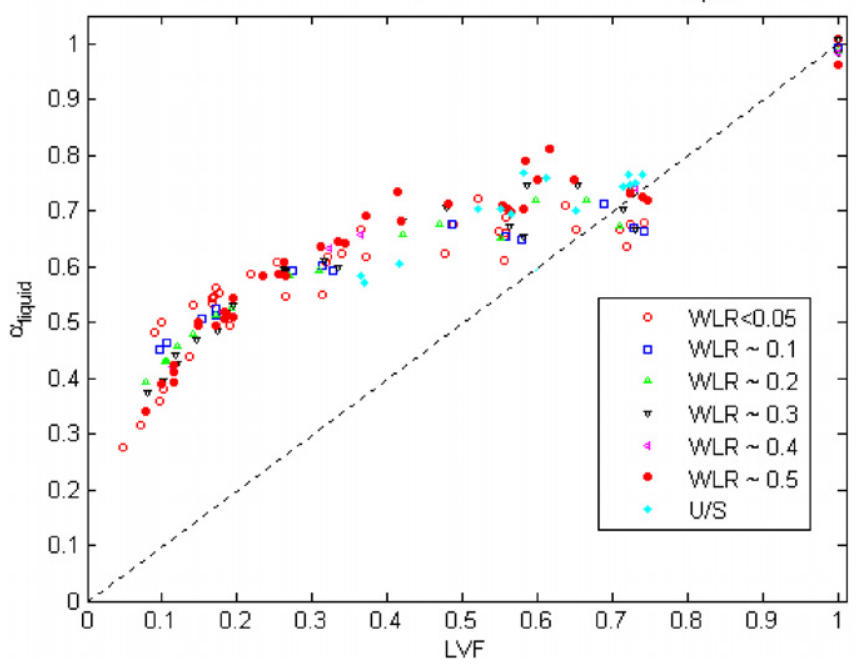

Figure 14. Plotted versus LVF, the time-average liquid holdup $\alpha_{\text {liquid }}$ estimated by the permittivity of mixture from opposite-electrode data using equations (13) and (14). Swirl flow with WLR up to $50 \%$. Ultrasound (U/S) sensor measured liquid holdup data also shown.

where $x$ is an empirical parameter for the gas-liquid annulartype distribution (including with $\mathrm{GVF}=0$ ).

The time-average liquid holdups derived from the permittivity of the mixture from the cross-pipe data measured by ECT are shown in figure 14, indicating that the liquid holdup increases with the increase in the liquid volume fraction (note that LVF $=1-\mathrm{GVF}$ ), and good agreement with some of the liquid holdups measured by ultrasound sensors at relatively high LVFs.

The model-based algorithm described in section 2.3 is an alternative method to derive both WLR and liquid-layer thickness $h$ (hence the liquid holdup) of horizontal swirl

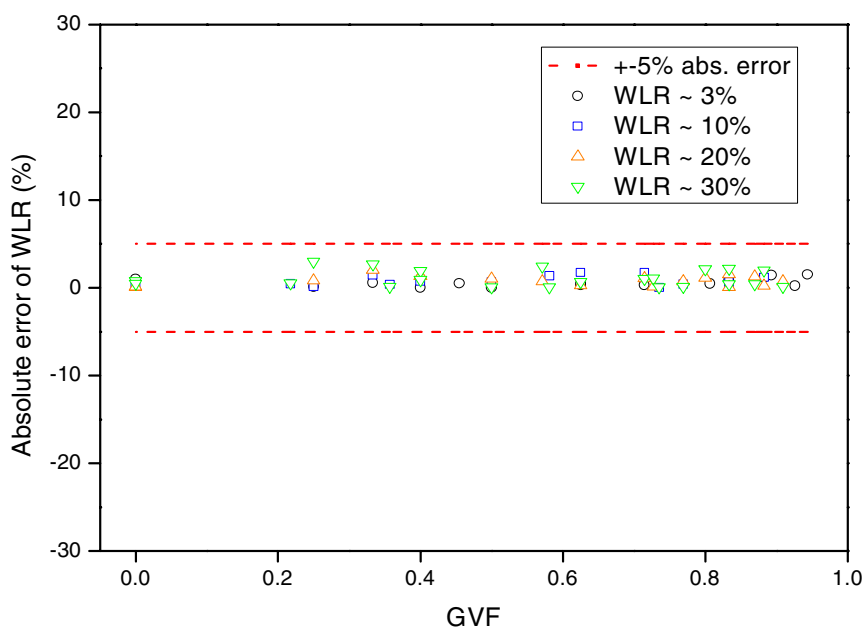

(a) WLR

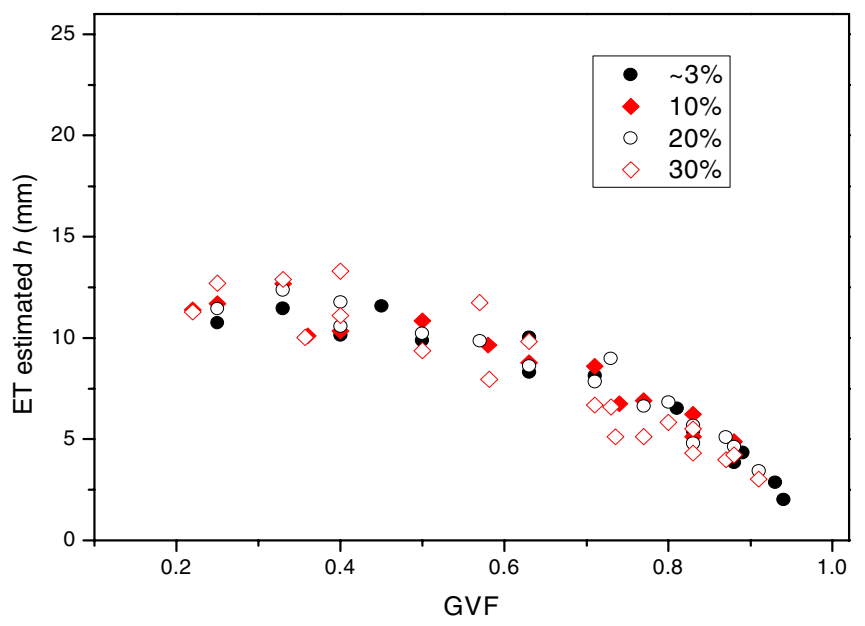

(b) Thickness of liquid layer

Figure 15. Model-based time-average results of liquid-layer WLR and thickness of flows by ECT.

gas/oil/water oil-continuous flows. A fast LBP algorithm can provide an initial estimate of the flow parameters for the use with the model-based iterative algorithm as shown in figure $3(b)$. Due to the dynamic nature of gas-liquid annular flows (see figure 12), the instantaneous capacitance measurements can be used to quantitatively deduce the WLR from liquid-rich data and the time-varying $h$ using the modelbased algorithm. Figure 15(a) shows the resulting timeaverage WLR, with an error of less than $\pm 5 \%$, for WLR up to $35 \%$ and GVF up to $95 \%$. Figure $15(b)$ shows the time average $h$. Consistent with the results in figure 14, $h$ decreases with the increase in the GVF; at GVF $>0.9, h$ can be less than $3 \mathrm{~mm}$.

\section{Conclusions and discussion}

This paper has described the measurement of gas/oil/water oil-continuous pre-conditioned annular flows by ECT together with the model-based image reconstruction algorithm. For a well-mixed oil-water (i.e. GVF $=0$, oil-continuous) swirl flow, the WLR can be estimated to be within $\pm 3 \%$ absolute, 
from ECT measurements. A new quantitative approach to interpretation of the permittivity of mixture measured from the normalized capacitance has been provided. This has facilitated a quantitative estimate of the WLR and a quantitative image reconstruction of the permittivity distribution over the crosssection of a pipe. For oil-continuous flows tested in TUV-NEL, satisfactory results of the WLR and liquid holdup are achieved for the horizontal swirl (annular) flows, where oil and water appear to be always well mixed into a homogeneous liquid phase. By utilizing the fast measurement of the permittivity of the liquid-rich slugs from multi-view measurements, the WLR of the liquid phase can be estimated to be within $\pm 5 \%$ for GVF up to $\sim 90 \%$, within the WLR measurement range of 0 $35 \%$. With the use of appropriate gas-liquid dielectric mixing model(s) developed in this work, the fast measurement of the permittivity of liquid-liquid and gas-liquid mixture permits the determination of the instantaneous liquid holdup and its time average. The multi-view instantaneous permittivity of mixture and spatial average has resulted in a liquid holdup potentially more immune to the instantaneous eccentric gas distribution.

\section{Acknowledgments}

The authors would like to thank the Technology Strategy Board of the UK for financially supporting this work under the Technology Programme (TP/8/OIL/6/I/Q2521G). The Technology Strategy Board is a business-led government body which works to create economic growth by ensuring that the UK is a global leader in innovation. Sponsored by the Department for Business, Innovation and Skills (BIS), it brings together business, research and the public sector, accelerating the development of innovative products and services to meet market needs, tackle major societal challenges and help build the future economy. TUV-NEL is also thanked for providing the test facilities and working as a project partner.

\section{Q4 References}

Falcone G, Hewitt G F and Alimonti C 2010 Multiphase Flow Metering (Developments in Petroleum Science vol 54) (Amsterdam: Elsevier)

Huang S M, Plaskowski A, Xie C G and Beck M S 1989 Tomographic Imaging of two-component flow using capacitance sensors J. Phys. E: Sci. Instrum. 22 173-7

Isaksen O, Dico A S and Hammer E 1994 A capacitance-based tomography system for interface measurement in separation vessels Meas. Sci. Technol. 5 1262-71

Isaksen O and Nordtved J E 1993 A new reconstruction algorithm for process tomography Meas. Sci. Technol. 4 1464-75

Ismail I, Gamio J C, Bukhari S F A and Yang W Q 2005 Tomography for multi-phase flow measurement in oil industry Flow Meas. Instrum. 16 145-55

Norwegian Society for Oil and Gas Measurement 2005 Handbook of Multiphase Flow Metering

Rajan V S V, Ridley R K and Rafa K G 1993 Multiphase flow measurement techniques-a review J. Energy Resour. Technol. 115 151-61

Ross A, Glen N, Bolton G and Qiu C H 2010 Use of dual-modality tomography for complex flow visualisation 9th Int. South East Asia Hydrocarbon Flow Measurement Workshop (Kuala Lumpur, Malaysia, 2-4 March)

Wang H G, Senior P, Mann R and Yang W Q 2009 Online solids moisture measurement and optimum control of fluidised bed dryer Chem. Eng. Sci. 64 2893-902

Xie C G 2006 Measurement of multiphase flow water fraction and water-cut 5th Int. Symp. on Meas. Technol. for Multiphase Flows (Macau, China, 10-13 Dec.) (AIP Conf. Proc. vol 914) 2007 pp 232-9

Xie C G, Atkinson I and Lenn C 2007 Multiphase flow measurement in oil and gas production Proc. 5th World Congress on Industrial Process Tomography (Bergen, Norway, 3-6 Sept.) pp 723-36

Xie C G, Huang S M, Hoyle B S, Thorn S, Lenn C P, Snowden D and Beck M S 1992 Electrical capacitance tomography for flow imaging: system model for development of image reconstruction algorithms and design of primary sensors $I E E$ Proc. G 139 89-98

Yang W Q and Byars M 1999 An improved normalisation approach for capacitance tomography Proc. 1st World Congress on Industrial Process Tomography (Buxton, UK, 14-17 April) pp 215-8

Yang W Q, Chondronasios A, Nattrass S, Nguyen V T, Betting M, Ismail I and McCann H 2004 Adaptive calibration of a capacitance tomography system for imaging water droplet distribution Flow Meas. Instrum. 15 249-58

Yang W Q and Peng L H 2003 Image reconstruction algorithms for electrical capacitance tomography Meas. Sci. Technol. $14 \mathrm{R} 1-\mathrm{R} 13$

Yang W Q, Stott A L, Beck M S and Xie C G 1995 Development of capacitance tomographic imaging systems for oil pipeline measurements Rev. Sci. Instrum. 66 4326-32

Yang W Q and York T A 1999 New AC-based capacitance tomography system IEE Proc.-Sci. Meas. Technol. $14647-53$ 


\section{QUERIES}

Page 1

Q1

Author: Please be aware that the colour figures in this article will only appear in colour in the Web version. If you require colour in the printed journal and have not previously arranged it, please contact the Production Editor now.

\section{Page 2}

Q2

Author: 'fluid mixture' or 'mixture fluid'? Please check throughout.

\section{Page 5}

Q3

Author: The sense of the sentence 'For a flow with a lower GVF ...' seems to be unclear. Please check.

\section{Page 12}

Q4

Author: Please check the details for any journal references that do not have a blue link as they may contain some incorrect information. Pale purple links are used for references to arXiv e-prints.

Q5

Author: Please provide author names in reference 'Norwegian Society for Oil and Gas Measurement 2005'.

Q6

Author: Please provide page range in reference 'Ross et al 2010 '. 